Retinal and Choroidal Imaging Update

\title{
Fundus autofluorescence imaging Fundamentals and clinical relevance
}

Yasir J. Sepah, MBBS ${ }^{\text {a }}$; Abeer Akhtar, B.S. ${ }^{\text {bcc }}$; Mohammad Ali Sadiq, MD ${ }^{\text {a }}$ Y Yamama Hafeez, B.S. ${ }^{\text {b }}$; Humzah Nasir, B.S. ;

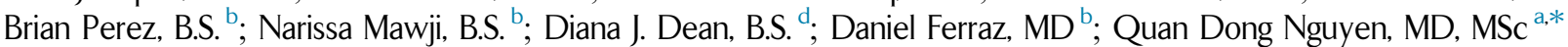

\begin{abstract}
Fundus autofluorescence (FAF), a relatively new imaging modality, focuses on the fluorescent properties of pigments in the retina to generate images that help us view various disease processes from a different perspective. It aids us in the understanding of the pathophysiology of different retinal disorders. Recently, FAF imaging is being used commonly to help us in the diagnosis, prognosis as well as in determining the treatment response of various retinal disorders. It generates an image based on the distribution pattern of a fluorescent pigment called lipofuscin. Knowing the distribution pattern of lipofuscin in the normal retina is key to understanding an FAF image representing a retinal pathology. Like most other imaging modalities, FAF comes with its own limitations, taking steps to overcome these limitations will be of utmost importance in using this imaging modality to its fullest potential.
\end{abstract}

Keywords: Fundus autofluorescence, Retina, Imaging

(C) 2014 Production and hosting by Elsevier B.V. on behalf of Saudi Ophthalmological Society, King Saud University. http://dx.doi.org/10.1016/j.sjopt.2014.03.008

\section{Introduction}

Fundus autofluorescence (FAF) is a relatively new, non-invasive imaging modality that has been developed over the past decade. The FAF images are obtained through the use of confocal laser scanning ophthalmoscopy (cSLO). It uses the fluorescent properties of lipofuscin to generate images that provide information beyond that is acquired by utilizing more conventional imaging methods such as fluorescein angiography, fundus photography, and regular optical coherence tomography (OCT). FAF has been an area of interest in ophthalmic research for over 40 years. However, it has only recently become clinically relevant because of various important technological advances. FAF has proved to be helpful in understanding the pathophysiological mechanisms, diagnostics and identification of predictive markers for disease progression, and for monitoring of novel therapies.

\section{Principle of auto-fluorescence imaging and interpretation of FAF Images}

\section{Retinal pigment epithelium (RPE) and lipofuscin}

RPE is a single layer of polygonal shaped cells, which separates the choroid from the neurosensory retina. This epithelial layer plays a critical role in the normal functioning of the retina. It is responsible for phagocytosis and lysosomal

Received 6 February 2014; received in revised form 26 February 2014; accepted 13 March 2014; available online 24 March 2014.

a Ocular Imaging Research and Reading Center, Stanley M. Truhlsen Eye Institute, University of Nebraska Medical Center, Omaha, NE, USA

${ }^{b}$ Retinal Imaging Research and Reading Center, Wilmer Eye Institute, Johns Hopkins University, Baltimore, MD, USA

c Harvard Medical School, Cambridge, MA, USA

d Creighton School of Medicine, Omaha, NE, USA

* Corresponding author. Address: Stanley M. Truhlsen Eye Institute, University of Nebraska Medical Center, 985540 Nebraska Medical Center, Omaha, NE 68198-5540, USA. Tel.: +1 (402) 5594276.

e-mail address: quan.nguyen@unmc.edu (Q.D. Nguyen).
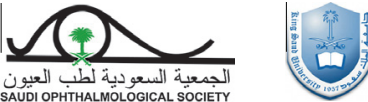

Peer review under responsibility of Saudi Ophthalmological Society, King Saud University 
breakdown of pigmented outer segments of photoreceptors, which allows the renewal process necessary to maintain photoreceptor excitability. Over the course of a lifetime, each RPE cell will phagocytose 3 billion outer segments. ${ }^{1}$ With aging, incomplete or partial breakdown of these segments in the post-mitotic RPE cells causes the accumulation of lipofuscin (LP). Lipofuscin is composed of several different molecules, most important of which is A2E ( $N$-retinyl- $N$-retinylidene ethanolamine) (Fig. 1). A2E is not recognized by lysosomal enzymes and therefore is incompletely broken down and accumulates in the lysosomes. An increased accumulation of this degraded material in the lysosomal compartment of the RPE cells is considered a hallmark of the aging process in the eye. In fact, a quarter of the RPE cytoplasm is composed of lipofuscin and melano-lipofuscin in persons over the age of seventy. Excessive lipofuscin deposition is considered pathologic and is associated with visual loss. There are significant clinical and experimental lines of evidence demonstrating that accumulation of lipofuscin above a certain threshold can cause functional loss of cells and lead to apoptosis.

Another component of lipofuscin, a toxic aldehyde known as all-trans retinal, is produced in the outer segments of the photoreceptor when exposed to light. Photoreceptors lack cis-trans isomerase function for retinal and are unable to regenerate all-trans-retinal into 11-cis-retinal after transduction of light energy (Fig. 2) into electrical impulses. ${ }^{21}$ The excess all-trans-retinal accumulates within the photoreceptor, forming bisretinoids which upon oxidation contribute to lipofuscin production. ${ }^{20}$

\section{Typical findings in the retina using fundus autofluorescence}

FAF images demonstrate a spatial distribution corresponding to the intensity of the signal emitted, where dark pixel values correspond to low intensities of emission and bright pixel values correspond to high intensities of emission. ${ }^{1}$ The naturally occurring autofluorescence of the

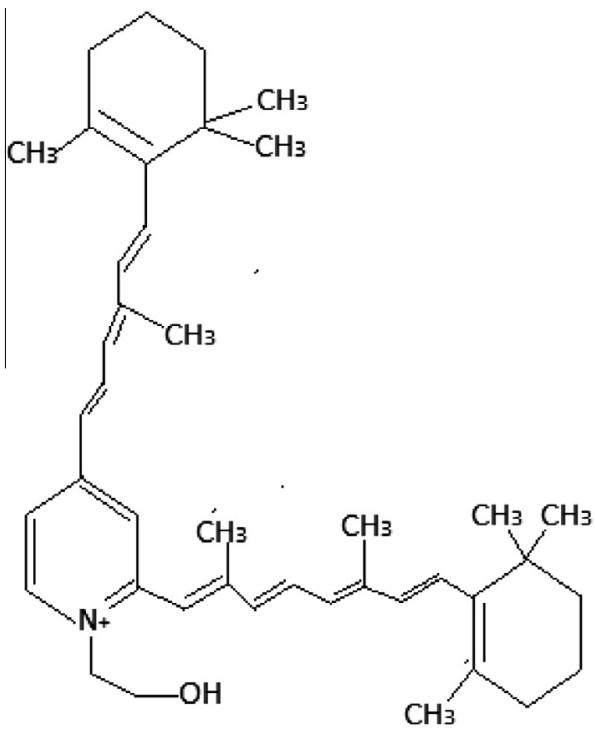

Figure 1. Chemical structure of A2E. (N-retinyl- $N$-retinylidene ethanolamine). ocular fundus is known to be of low intensity such that the distribution of FAF in normal eyes demonstrates a consistent pattern in which the optic nerve head typically appears dark due to the absence of lipofuscin in this area. ${ }^{1}$ Retinal vessels are characterized by a reduced FAF signal due to the absorption by blood. ${ }^{1}$ FAF signal is also reduced in the macular area, particularly around the fovea due to the absorption of the luteal pigment. ${ }^{1}$ It can be noted that even though the signal in the parafoveal area tends to be higher, it presents with a relatively reduced intensity when compared to the background signal in the more peripheral areas of the retina. This observation is thought to be caused by an increased concentration of melanin, and decreased concentration of lipofuscin granules in central RPE cells. ${ }^{1}$

However, the distinction between the optic disk and the macula is reversed if the wavelength of the excitation source is changed (e.g. devices using blue light will give you the former pattern on the FAF images while devices using the green light will give you the latter) (Fig. 3). Green light FAF is relatively new and has not been available commercially until recently. Hence there is a lack of information as to how various diseased retinas would appear when scanned with a device utilizing a green light as the fluorophore excitation source.

\section{Interpretations of the fundus autofluorescence images}

Fundus autofluorescence imaging is used to record fluorescence that may occur naturally in ocular structures or as a byproduct of a disease process. This technique allows the topographic mapping of lipofuscin distribution in the RPE. ${ }^{1}$ The intensity portrayed by FAF corresponds to the accumulation of lipofuscin, which increases with aging, RPE cell dysfunction or an abnormal metabolic load on the RPE.

When evaluating an FAF image, any deviation from the normal should be thoroughly investigated to identify a possible cause. Reasons for a reduced FAF signal may include but are not limited to: RPE loss or atrophy, intraretinal fluid, reduction in RPE lipofuscin density, fibrosis or presence of luteal pigment. ${ }^{1}$ Causes for increased FAF signal may include but are also not limited to: drusen in the sub-pigmented epithelial space, excessive RPE lipofuscin accumulation, age-related macular degeneration or the occurrence of fluorophores anterior or posterior to the RPE cell monolayer. ${ }^{1}$ However, it is to be noted that the quality of the image may be affected by the opacity of the vitreous, lens, cornea or anterior chamber and thus influence the identification of abnormalities. Thinner areas of retina adhere to the "window effect" meaning that they exhibit increased autofluorescence otherwise known as hyperautofluorescence. ${ }^{2}$

\section{Clinical applications of fundus autofluorescence imaging}

Various histopathological studies have demonstrated the accretion of autofluorescent material and deposits in the RPE in various retinal dystrophies. Since FAF imaging enables the visualization of changes in lipofuscin distribution in the $\mathrm{RPE}^{3}$ it can be useful in providing information about retinal dystrophies in which the health of the RPE is an important factor. FAF imaging has proven to be useful in regard to understanding and providing new perspectives concerning 


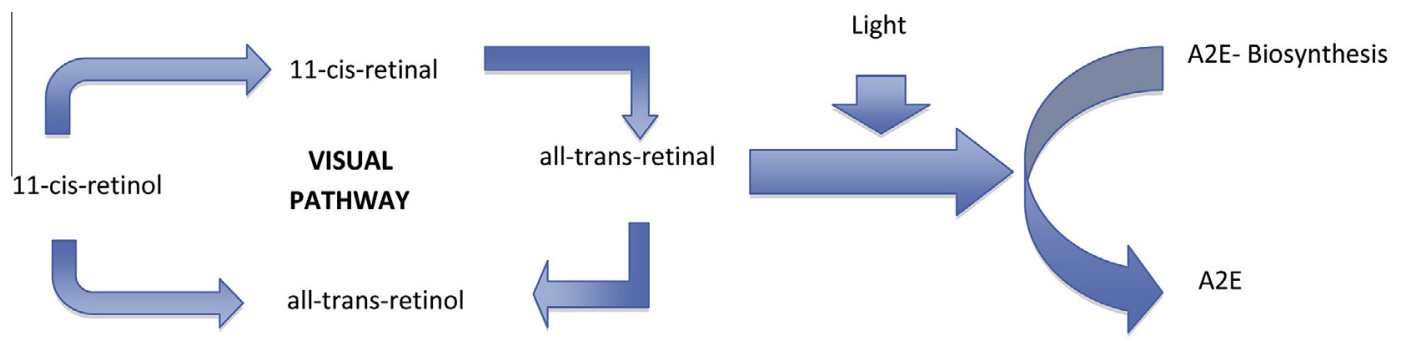

Figure 2. Biochemical pathway representing various steps involved in the metabolism of lipofuscin.
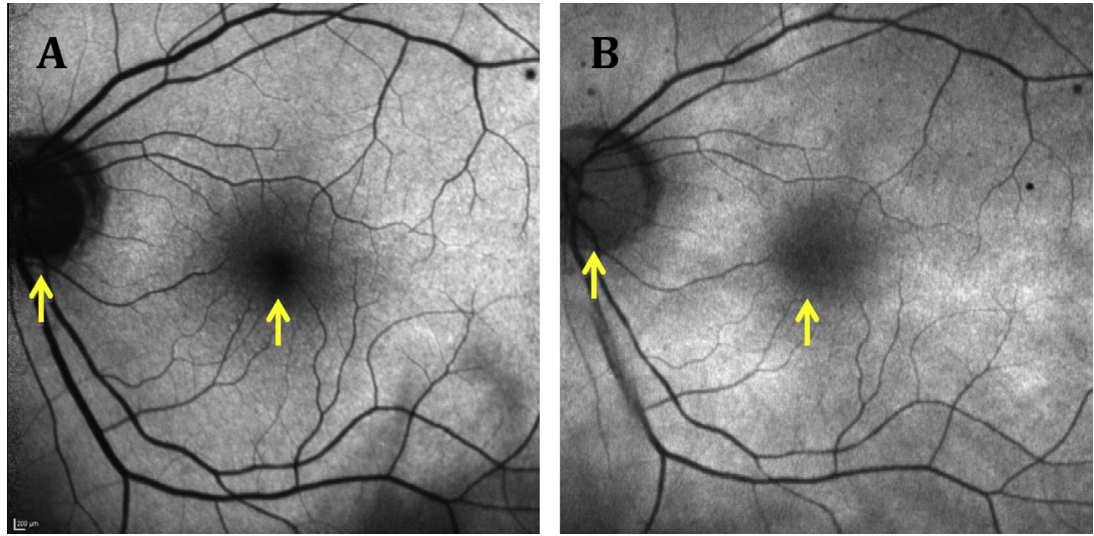

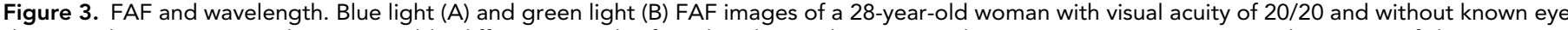

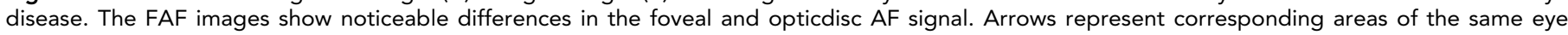
showing different AF signal when imaged with two different optical systems.

various pathophysiological mechanisms. FAF has also played a role in the monitoring of novel therapies of the retina and the identification of predictive markers for disease progression. ${ }^{1}$ FAF allows for non-invasive mapping and documentation of metabolic change by noting level of fluorescence, providing information that may otherwise not be clinically detectable. FAF findings have aided physicians in the identification of early retinal disease stages as well as phenotyping in retinal dystrophies such as retinitis pigmentosa. Additionally, FAF has recently been added to the imaging studies recommended by the National Eye Institute (National Institutes of Health, USA) to investigate and follow patients suspected of chloroquine retinopathy that results in devastating visual loss (Fig. 4). The future identification of high-risk characteristics or biomarkers can be useful in following patients and for performing clinical trial interventions in certain cases.

\section{Age-related macular degeneration}

Age-related macular degeneration (AMD) is a progressive chronic pathology which is considered the most common cause of legal blindness in industrialized countries. ${ }^{4}$ Both environmental and genetic risk factors have been observed to influence the development of AMD. ${ }^{1,5}$ The presence of drusen in color fundus photography is considered pathognomonic of AMD. ${ }^{6}$

Since the accumulation of lipofuscin in post-mitotic RPE cells has been postulated to play an important role in the pathogenesis of $A M D, 5,7$ the monitoring of the metabolic integrity of the RPE with FAF imaging is useful to the clinician monitoring disease progression in AMD. Increased FAF is seen due to excessive accumulation of lipofuscin within the lysosomal compartment of RPE and decreased FAF is characteristic in patients with geographic atrophy (GA) due to absence of lipofuscin granules in the RPE ${ }^{6}$ (Figs. 5 and 6).

FAF has been described as a prognostic marker of GA. While FAF has been described as a marker for progression of the disease, some authors did not find any correlation between abnormal FAF and progression of GA. ${ }^{7}$ Nevertheless, there is evidence that increased autofluorescence in the $500 \mu \mathrm{m}$ margin around areas of GA could be used to distinguish between fast and slow progression of the disease. ${ }^{8}$

Different patient classification systems have been proposed for describing FAF imaging findings in AMD. In 2005, Bindewald et al. proposed a classification to describe FAF as focal, banded, patchy and diffuse in patients with GA. ${ }^{9}$ Additionally another kind of classification in patients with early AMD was proposed by Bindewald to describe early AMD as normal, minimal change, focal, increased, patchy, linear, lacelike, reticular and speckled. ${ }^{10}$ In 2002, Lois had proposed to describe FAF as focal, increased, reticular, combined and homogeneous. ${ }^{7,11}$ As distinctions between the classifications in these studies is hazy, standardized terminology is greatly needed to describe pathologic patterns of FAF.'

Midena et al. studied the relationship between microperimetry and FAF of the macular region with drusen and pigment abnormalities in early AMD and found that microperimetry and FAF can be helpful for monitoring the progression of AMD before changes in visual acuity are observed. ${ }^{12}$

\section{Retinal artery occlusion}

Retinal artery occlusions are blockages in the small arteries that carry blood to the retina. In most patients, the central 

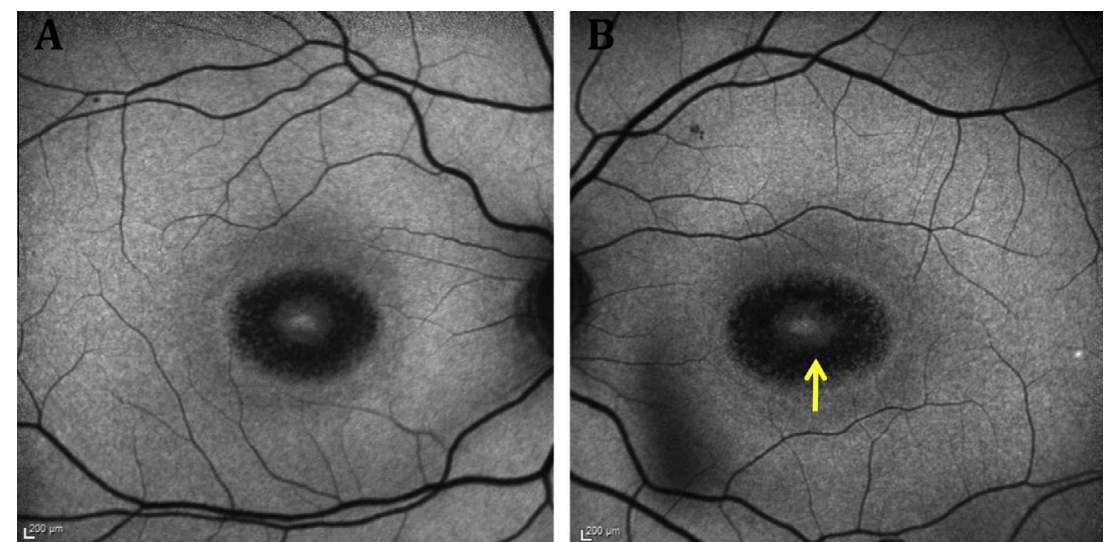

Figure 4. Chloroquine maculopathy. Fovea surrounded by circular band of hypoautofluorescence with OS (B) effected to a greater extent than OD (A). Arrows point to the macular lesion of chloroquine toxicity.
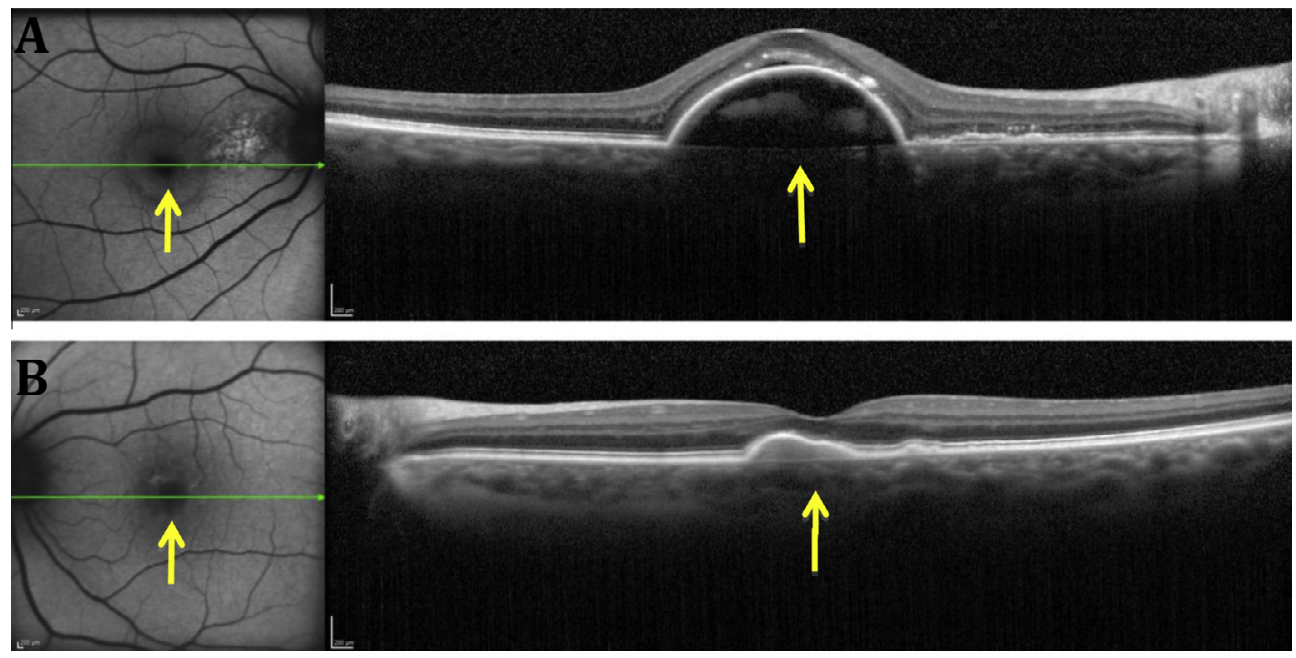

Figure 5. Neovascular AMD, right eye (A) with more advanced disease compared to left eye (B). Fovea surrounded by a band of hypoautofluorescence corresponding to the site of neovascularization shown by OCT. Both arrows are pointing toward the disease process surrounding the fovea.
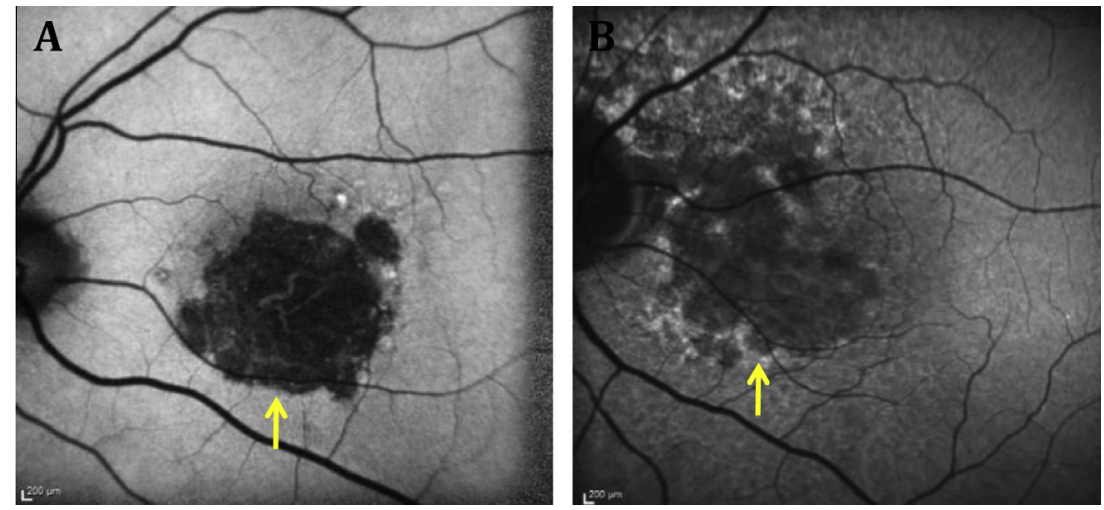

Figure 6. GA. (A) Well-circumscribed area of hypoautofluorescence. (B) Area of hypoautofluorescence surrounded at the edges by hyperautofluorescence as shown by the arrow. Arrows point toward the boundary of the GA lesion.

retinal artery provides the only blood supply for the retina. ${ }^{13}$ Historically, complications such as occlusions have generated poor visual outcomes, as a vast majority do not regain vision. ${ }^{13}$ However, FAF has proven to be a useful tool in pointing out areas of retinal artery occlusion. Occlusions inhibit proper autofluorescence of the retinal pigment epithelium due to increased thickness resulting in a decrease in FAF. ${ }^{2}$ Such property allows the identification of areas of the retina that are ischemic and of lower AF intensity (Fig. 7). Hence, the technique can be used to objectively assess response to therapy or a natural reduction in the severity of the disease. It has been shown that the level of 


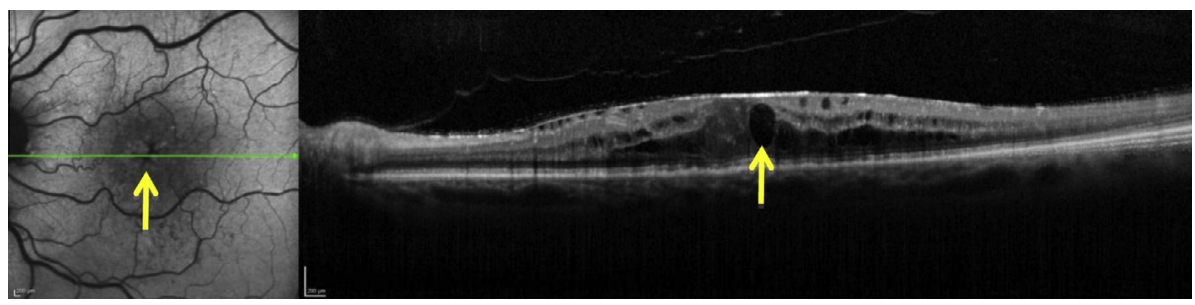

Figure 7. A diffuse area of hypoautofluorescence area involving the fovea and the surrounding area in an eye with cystic macular edema associated with central retinal vein occlusion. Arrow on the left side of the image is pointing toward the area of hypoautofluorescence and arrow on the right side is pointing toward cystic changes in the macula.

FAF exhibited in a particular area of the retina is proportional to the thickness of that area, thus proving useful in imaging retinal artery occlusions. In theory, FAF would allow for better measurement of the progression or regression of the disease as compared to the current standards of measurement, which include visual acuity.

\section{White dot syndromes}

White dot syndromes refer to a constellation of rare inflammatory disorders affecting the retina, retinal pigment epithelium and choroid. ${ }^{14,15}$ Diagnosis of a white dot syndrome in a patient is often challenging due to the similarities in the presentation among different disorders. Fundus autofluorescence could prove a valuable diagnostic tool in helping with this diagnostic challenge. ${ }^{16}$ Not all forms of white dot syndromes actually produce white dots, but they all do express lesions in the fundus. ${ }^{15}$ The ones that do produce white dots, such as multifocal choroiditis and punctate inner choroidapathy, may exhibit hyper- or hypoautofluorescence on FAF in the lesions and the areas around the dots (Fig. 8). ${ }^{17}$ In a study conducted by Yeh et al. it was found that FAF hypofluorescence around the foveal area was positively correlated with poorer visual acuity. ${ }^{16}$ Such correlation provides important evidence, as hypofluorescence caused by inflammation of the RPE is associated with decreased vision.

Autofluorescence as a diagnostic tool may therefore provide more accurate information than visual acuity on the status of a patient with white dot syndrome, as there are many other impediments that can affect visual acuity.

\section{Diabetic retinopathy}

In diabetic retinopathy (DR), the mechanism of accumulation of lipofuscin in the retina is different as compared to AMD. Lipofuscin is composed of different products of peroxidation of lipids and proteins; for this reason, lipofuscin is considered a marker of oxidative processes in the retina. ${ }^{18}$ $\mathrm{Xu}$ et al. showed that accumulation of lipofuscin in mice might be found in a greater proportion in microglia than in the RPE. ${ }^{19}$ As is currently understood, the pathophysiologic process of DR activates microglia, allowing the activation and formation of oxidative by-products and therefore formation of lipofuscin granules, which can be detected by FAF imaging (Fig. 9). ${ }^{18,19}$ Currently, not a lot of work has been done to exploit the usefulness of FAF imaging in DR patients. However, based on animal studies and small clinical studies, FAF has shown tremendous potential and it is expected to become an important imaging technique in caring for patients with DR.

\section{Limitations and future directions}

The use of FAF to map natural or pathological presence of fluorophores has influenced our understanding and interpretation of various kinds of retinal diseases. The future of this imaging technique may allow further understanding of retinal diseases as certain aspects of this technique begin to be better understood. It may also be used in patients with retinal dystrophies to access the potential functional preservation of the outer retina, which would therefore have implications in monitoring or evaluation of treatments for this in the
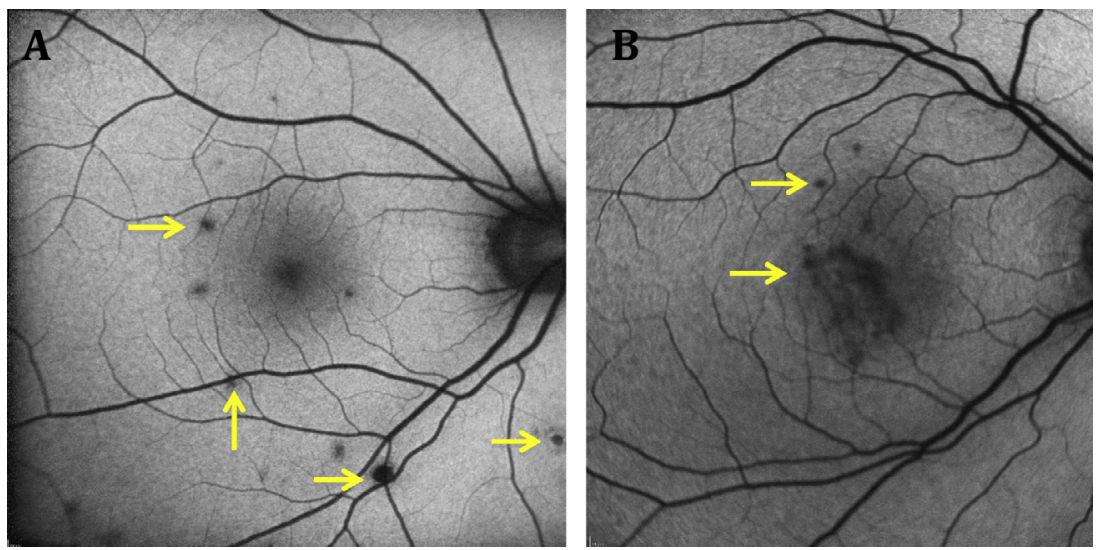

Figure 8. Well-circumscribed lesions of multifocal choroiditis within and outside the arcades in a stable eye showing hypoautofluorescence (A). Similar lesions of PIC located within the arcades showing hypoautofluorescence (B). 

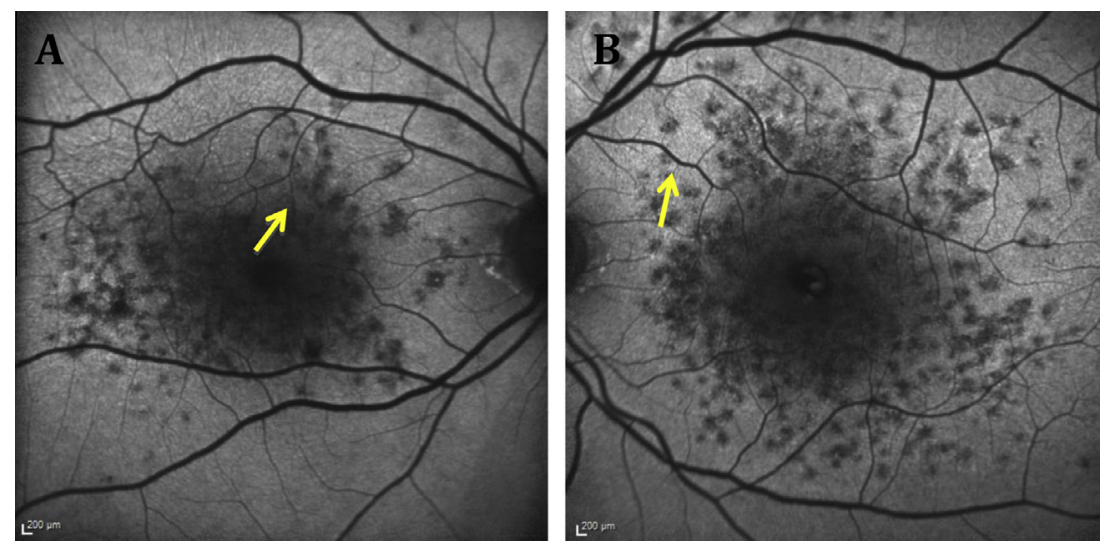

Figure 9. Numerous small well-circumscribed areas of hypoautofluorescence, left eye (B) more than the right eye (A) in eyes with diabetic macular edema representing leakage from microaneurysms.

future. ${ }^{17}$ At present, most limitations arise from the fluorescent properties of other ocular structures, as well as the resolution of the image and the time taken to acquire a good quality image. ${ }^{1,16}$ The optical media and the lens, which may present confounding background noise, can influence the intensity of fluorescence coming from the retina. Improving the acquisition method of this technique can increase the resolution and reduce the background noise in the topographic mapping of fluorophores. ${ }^{1,16}$ With increasing age, fluorescent properties of the lens also change, which makes the uses of FAF in older patients more difficult to interpret. Presently, scanning laser ophthalmoscopy (SLO) addresses the limitations of reduced intensities of $\mathrm{AF}$ and the fluorescent crystalline lens; yet, this technique is limited by the optical properties of the eye. Therefore, further advancements in auto-fluorescence using SLO need to address these limitations in order to improve image resolution. ${ }^{1,16}$ In the attempt to reduce background noise and improve image contrast, new developments are currently being made. Such developments include real-time averaging, where mean images are obtained to effectively reduce background noise. In addition, fifty-five degree wide-field view image acquisition with the use of a new lens highlights recent developments in FAF. ${ }^{1}$ Thus, the applications of FAF most likely will remain protean, with future advancements confirming (or disproving) the use of this technology in the management of ocular diseases.

\section{Conflict of interest}

The authors declared that there is no conflict of interest.

\section{References}

1. Schmitz-Valckenberg $S$ et al. Fundus autofluorescence imaging: review and perspectives. Retina 2008;28(3):385-409.

2. Mathew R, Papavasileiou E, Sivaprasad S. Autofluorescence and highdefinition optical coherence tomography of retinal artery occlusions. Clin Ophthalmol 2010;4:1159-63.

3. Boon CJ et al. Fundus autofluorescence imaging of retinal dystrophies. Vision Res 2008;48(26):2569-77.
4. Schmitz-Valckenberg $S$ et al. Fundus autofluorescence and progression of age-related macular degeneration. Surv Ophthalmo 2009;54(1):96-117.

5. Lim LS et al. Age-related macular degeneration. Lancet 2012;379(9827):1728-38.

6. Smith RT et al. Autofluorescence characteristics of early, atrophic, and high-risk fellow eyes in age-related macular degeneration. Invest Ophthalmol Vis Sci 2006;47(12):5495-504.

7. Hopkins J, Walsh A, Chakravarthy U. Fundus autofluorescence in agerelated macular degeneration: an epiphenomenon? Invest Ophthalmol Vis Sci 2006;47(6):2269-71.

8. Bearelly $\mathrm{S}$ et al. Use of fundus autofluorescence images to predict geographic atrophy progression. Retina 2011;31(1):81-6.

9. Bindewald $A$ et al. Classification of abnormal fundus autofluorescence patterns in the junctional zone of geographic atrophy in patients with age related macular degeneration. $\mathrm{Br} J$ Ophthalmo 2005;89(7):874-8.

10. Bindewald $A$ et al. Classification of fundus autofluorescence patterns in early age-related macular disease. Invest Ophthalmol Vis Sci 2005;46(9):3309-14

11. Lois $\mathrm{N}$ et al. Fundus autofluorescence in patients with age-related macular degeneration and high risk of visual loss. Am J Ophthalmol 2002;133(3):341-9.

12. Midena $\mathrm{E}$ et al. Microperimetry and fundus autofluorescence in patients with early age-related macular degeneration. $\mathrm{Br} J$ Ophthalmol 2007;91(11):1499-503.

13. Sharma S. Ophtha problem. Central retinal arterial occlusion. Can Fam Physician 1997;43, p. 1513, 1520.

14. Abu-Yaghi NE et al. White dot syndromes: a 20-year study of incidence, clinical features, and outcomes. Ocul Immunol Inflamm 2011:19(6):426-30.

15. Matsumoto Y, Haen SP, Spaide RF. The white dot syndromes. Compr Ophthalmol Update 2007;8(4):179-200, discussion 203-4.

16. Yeh $\mathrm{S}$ et al. Fundus autofluorescence imaging of the white dot syndromes. Arch Ophthalmol 2010;128(1):46-56.

17. Penha FM et al. Fundus autofluorescence in multiple evanescent white dot syndrome. Case Rep Ophthalmol Med 2011;2011:807565.

18. Vujosevic $S$ et al. Diabetic macular edema: fundus autofluorescence and functional correlations. Invest Ophthalmol Vis Sci 2011;52(1):442-8.

19. $\mathrm{Xu} \mathrm{H}$ et al. Age-dependent accumulation of lipofuscin in perivascular and subretinal microglia in experimental mice. Aging Cell 2008;7(1):58-68.

20. Lambris JD, Adamis Anthony P. Inflammation and retinal disease: complement biology and pathology. In: Advances in experimental medicine and biology, vol. 703. New York, NY: Springer; 2010, pp. 63-74, Print.

21. Strauss Olaf. The retinal pigment epithelium in visual function. Physiol Rev 2005;85:845-81. http://dx.doi.org/10.1152/physrev.00021.2004. 